\title{
Some observations on electroencephalograms of myoclonus epilepsy
}

\author{
I. KADOBAYASHI, G. UKIDA, AND I. KAWAI ${ }^{1}$
}

From the Department of Psychiatry, Kyoto Prefectural University of Medicine, Kawaramachi-hirokoji, Kamigyo-ku, Kyoto, Japan

SUMMARY Electroencephalographic observations of four cases of myoclonus epilepsy are reported. A feature in EEG findings was that spikes became more prominent in the later stage of illness. Photic stimulation had a distinct activating effect on producing paroxysms, and an increase in number of paroxysms depended not on the frequency of stimulus but on the intensity. A sleep EEG showed ill-formed humps and sigma rhythm with infrequent paroxysms. It is suggested that these observations might be due to a disturbance in the function of subcortical structures.

Electroencephalograms (EEG) of progressive myoclonus epilepsy first described by Unverricht $(1891,1895)$ have been reported by previous authors (Grinker, Serota, and Stein, 1938; Gastaut and Rémond, 1952; Harriman and Millar, 1955) and EEGs in series were described as constant during the course of illness (Van Heycop Ten Ham and De Jager, 1963). It is well known that photic stimulation is effective in producing myoclonic jerks and paroxysmal waves, but knowledge of its mechanism remains incomplete. Furthermore, while myoclonic jerks are absent during sleep, no sleep-recording has been reported. We have had an opportunity to record EEGs of four cases of myoclonus epilepsy, including sleep EEGs, and have attempted to gain some insight into the mechanism underlying the electroencephalographic findings.

CASE 1

M.T., born 7 May 1914, was a 54 year old woman whose father and son had suffered from the same illness. At the age of 25 years she experienced her first generalized convulsive seizure, which was preceded by myoclonic jerking movements of the upper extremities. Seizures occurred at first three times a year and myoclonic jerks were not seen without a seizure. From the age of 30 years she had a seizure a month, and myoclonic jerks before seizures became more pronounced and their duration longer. From the age of 38 years, independently of seizures, slight jerking movements of the upper extremities continuously occurred except during sleep, and later became more

'Present address: Department of Psychiatry, Kyoto University, Sakyo-ku, Kyoto, Japan. severe. From the age of 48 years she became bedridden for her massive myoclonic jerks which were precipitated by light and attention. In June 1966 she experienced a long-lasting postictal twilight state for three days. Five months later the patient was admitted to the Kyoto Prefectural University Hospital. On examination she looked older than her years, and her myoclonic jerks were more marked in the upper extremities than in the lower extremities, trunk, neck, and tongue. Her gait was ataxic and her speech was dysarthric. Otherwise little abnormality was revealed on neurological examination. Findings from routine blood analysis, serum electrolytes, and cerebrospinal fluid were unremarkable. Pneumoencephalography showed a slight dilatation of the ventricles. Liver biopsy did not reveal Lafora bodies. She was grossly deteriorated intellectually and her IQ (WAIS) was 61 .

CASE 2

K.T., born 3 February 1938, was a 30 year old man, the first of five siblings, whose mother was the first case. His sister's EEG was flat (Kadobayashi, Ukida, Kita, and Iizuka, 1969). From the age of 27 years he had jerks of both hands once or twice a day. Jerks occurred often during reading papers and when strained. He also had attacks with sudden muscular spasms of the extremities. These attacks occurred during transition states from the waking state to sleep. On examination he was a tall, wellbuilt man with slight myoclonic jerking of the hands. Neurological examination was negative. There was no evidence of mental deterioration. Seizures were not perceptibly affected by anticonvulsant medication.

CASE 3

T.N., born 16 September 1941, was a 25 year old woman, 
the ninth of 10 siblings, whose younger brother suffered from the same illness. From the age of 14 years she had convulsive seizures, and jerking movements of the extremities and the trunk. The jerking movements were usually bilateral, but especially pronounced on the left side. At the age of 24 years speech and sight were suddenly impaired, but three months later she had recovered them on medication. From that time she had progressive difficulty in walking. In January 1967, the patient was admitted to the Kyoto University Hospital because of inability to walk. On examination there was an intermittent dysarthria and her gait was ataxic. Otherwise there was little abnormality on neurological examination.

CASE 4

S.U., born 18 December 1945, was a 19 year old boy with no known disposition to neurological diseases. Generalized convulsive seizures started at the age of 10 years. The seizures occurred twice a year. At 18 years of age he had massive myoclonic jerks of all muscles beginning with the right hand, while he remained conscious. From that time myoclonic jerks were seen every day. In August 1965 he was admitted to the Kyoto Prefectural University Hospital because of difficulty in walking. On examination he had continuous myoclonic jerking movements of all the voluntary muscles. There was ataxia and dysarthria. No pathological reflexes were elicited. Findings from routine blood analysis, serum electrolytes, and cerebrospinal fluid were unremarkable. Pneumoencephalography showed normal findings. The fundi were normal. In the hospital natural sunshine once induced a generalized convulsive seizure. His IQ was 68 . Afterwards he was successfully treated with anticonvulsants.

\section{RESULTS}

RESTING RECORD A series of EEGs was recorded in cases 1, 2, and 4 and a single EEG in case 3 .

EEG of case 1 In December 1966 when the patient was 52 years old, there was an irregular basic pattern with fast activity of 15 to $24 \mathrm{~Hz}$ (Fig. 1). This fast activity was marked in parietal and central areas with amplitudes of 40 to $90 \mu \mathrm{V}$, and sometimes changed into sharp waves. No $\alpha$-rhythm was discernible. Spikes and polyspikes frequently occurred in almost all leads, maximal in parietal areas. Less frequertly, atypical spike-wave complexes at 3 to $4 / \mathrm{sec}$ and high-voltage slow waves of 4 to $6 \mathrm{~Hz}$ with amplitudes up to $130 \mu \mathrm{V}$ appeared in almost all leads, less marked in occipital areas. Two other EEGs recorded 10 months and 11 months later were similar, though spike-wave complexes seldom appeared.

EEG of case 2 In March 1967, when he was 29 years old, there was a low-voltage irregular basic pattern with fast activity of 16 to $22 \mathrm{~Hz}$ with amplitudes of 20 to $35 \mu \mathrm{V}$ in parietal areas. Atypical spikewaves at 3 to $4 / \mathrm{sec}$ and high-voltage slow waves of

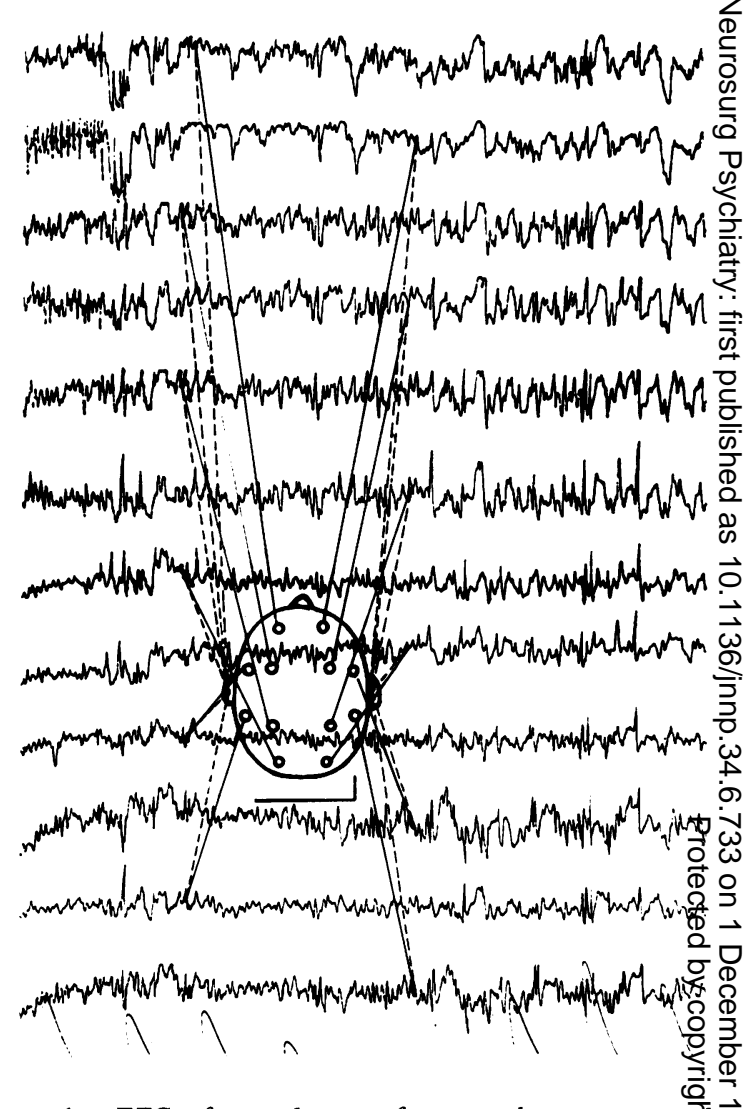

FIG. 1. EEG of case 1; note frequent sharp waves a $\overrightarrow{0}$ spikes with fast activity. Calibrations indicate $50 \mu \mathrm{V}$ and $\underset{ }{ }$ 1 sec in this and following Figures.

4 to $6 \mathrm{~Hz}$ with amplitudes of 100 to $150 \mu \mathrm{V}$ appeared $\frac{0}{1}$ in almost all areas, maximal in parietal areas. They often appeared asymmetrically. Their amplitudes $\bar{O}$ were usually greater on the right side, but sometimes vice versa. Opening of the eyes and external sudden voice resulted in spike-waves. Another EEG recorded one year later showed a similar background activity, though amplitudes of spike components of spikewaves were greater (Fig. 2).

EEG of case 3 In March 1967, at the age of $26 \%$ years, there was an irregular background activity with fast waves of 14 to $30 \mathrm{~Hz}$ with amplitudes of 을 20 to $40 \mu \mathrm{V}$ in parietal areas (Fig. 3). Against this $D$ background, there occurred brief transient paroxysms of spikes and polyspikes in almost all areas, $\bar{N}$ especially marked in parietal areas. High-voltage or slow waves of 3 to $4 \mathrm{~Hz}$ with amplitudes of 100 to $150 \mathrm{~N}$ $\mu \mathrm{V}$ were also visible in fronto-central areas. 


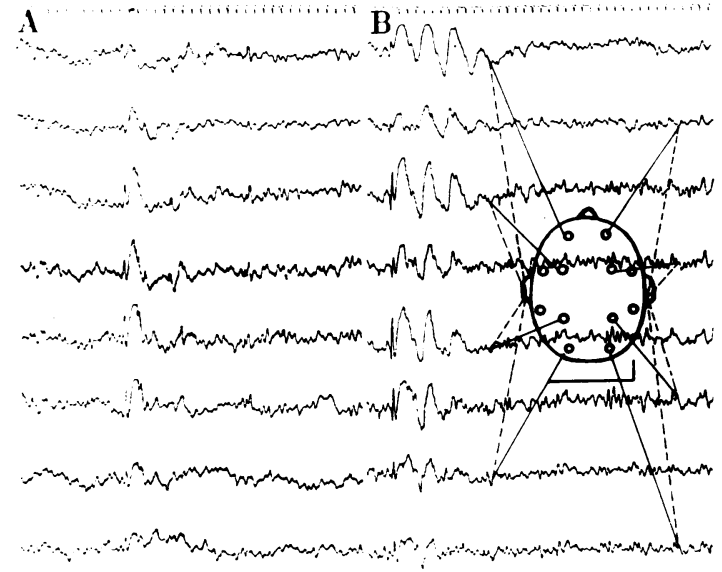

FIG. 2. EEG of case 2; note an increase in amplitude of spike components of spike-waves in the EEG recorded one year later. A. EEG recorded on 31 March 1967. B. EEG recorded on 13 March 1968.

EEG of case 4 In September 1965 there was an irregular basic pattern with normal-voltage slow waves of 6 to $8 \mathrm{~Hz}$. Sometimes fast activity of 17 to $23 \mathrm{~Hz}$ with amplitudes of 20 to $40 \mu \mathrm{V}$ appeared in centro-parietal regions. High-voltage slow waves of 3 to $6 \mathrm{~Hz}$ appeared in bursts with maximum voltage in frontal areas (Fig. 4). They often reached amplitudes of about $200 \mu \mathrm{V}$ and were followed by myoclonic jerks. Another EEG recorded one month previously was often interrupted by artefacts of

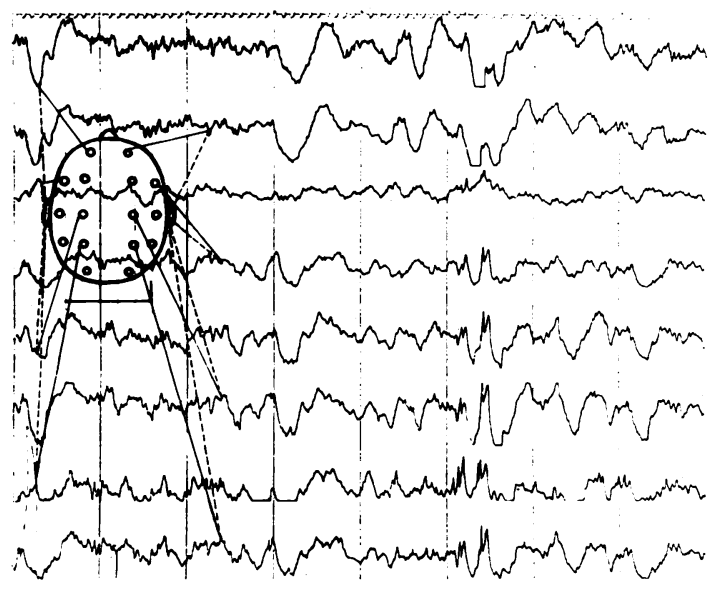

FIG. 3. EEG of case 3; note spikes maximal in parietal areas with high-voltage slow waves.

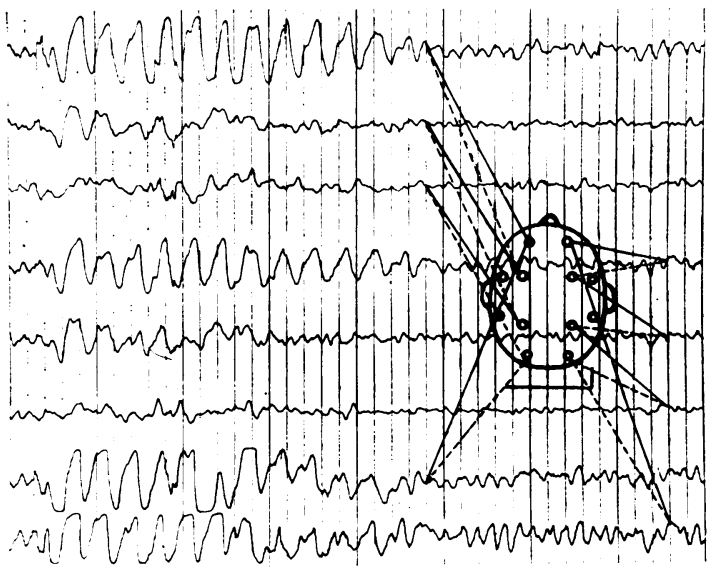

FIG. 4. EEG of case 4; note bursts of high-voltage slow waves.

massive myoclonus. It showed an irregular basic pattern with marked fast activity of 17 to $19 \mathrm{~Hz}$, and paroxysms of slow waves of 6 to $7 \mathrm{~Hz}$ and of small spikes.

PHOTIC ACTIVATION In all cases, photic stimulation had a distinct activating effect in producing ратохysms, but their occurrence was hardly dependent upon the frequency of light stimulus. In the second case, 6,8 , and $10 \mathrm{~Hz}$ stimulation resulted in spikes, while 3,14 , and $16 \mathrm{~Hz}$ stimulation provoked spikewaves. In the third case, frequencies of 5 and $14 \mathrm{~Hz}$ were strikingly effective, though a frequency of $10 \mathrm{~Hz}$ was less effective. In the first case, high fiequencies ( $c a .20 \mathrm{~Hz}$ and greater) were rather more effective than low frequencies. Van Heycop Ten Ham and De Jager (1963) also reported that photic stimulation, particularly at high frequencies, induced multiple spikes and spike-wave complexes. These facts suggest that the appearance of paroxysms might be related to photostimulus intensity rather than to frequency. We tried to observe an electroencephalographic change, varying distances between flash lamp and patient's eyes in a dark room. In order to avoid as many other factors as possible, the retina was stimulated with the same frequency ( 5 and $10 \mathrm{~Hz})$ for the same period $(10 \mathrm{sec})$ at intervals of five minutes and more. As was expected, in all cases paroxysms occurred more frequently, as the distance shortened-that is, the light became more intense. Figure 5 shows an example in the first case during $5 \mathrm{~Hz}$ photic stimulation. In this case photic stimulation produced spikes which were marked in parietal areas. The number of spikes of more than $70 \mu \mathrm{V}$ 


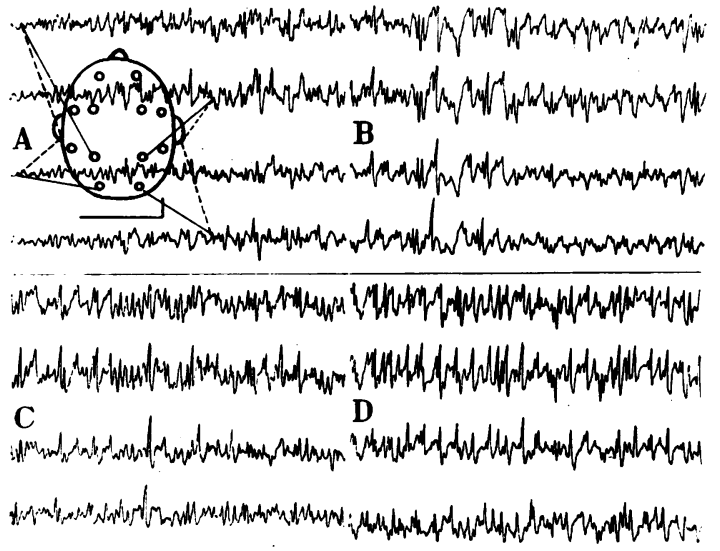

FIG. 5. An electroencephalographic change by photic stimulation with $5 \mathrm{~Hz}$, when the distance between flash lamp and patient's eyes was varied. A, B, C, and D indicate distances of $75,60,45$, and $30 \mathrm{~cm}$ respectively.

from the right parietal area for 10 seconds at different distances is shown in Table 1. As is shown in this Table, the number of spikes was nearly inversely proportional to the square of the distance-that is, directly proportional to light intensity.

\section{TABLE 1}

NUMBER OF SPIKES OF MORE THAN $70 \mu \mathrm{V}$ FROM RIGHT PARIETAL AREA DURING 5 HZ PHOTIC STIMULATION WITH DIFFERENT DISTANCES BETWEEN FLASH LAMP AND PATIENT'S EYES

\begin{tabular}{cc}
\hline Distance $(\mathrm{cm})$ & Number of spikes \\
\hline 30 & 32 \\
45 & 15 \\
60 & 7 \\
75 & 5 \\
\hline
\end{tabular}

SLEEP EEG In the first, second, and fourth case sleep EEGs were recorded, but the second and fourth case remained only in drowsy state. The first case alone reached a deep sleep. Figure 6 shows each stage of sleep EEG in the first case. In the drowsy state fast activity remained as before, whereas other activity was flattened. At times 4 to $7 \mathrm{~Hz}$ slow waves of moderate amplitude were mixed. There occurred sporadically spikes, sharp waves and diphasic positive-negative sharp waves in bilateral parietal, occipital, and temporal areas, in contrast with frequent paroxysms of spikes, polyspikes, and sharp waves in the awake state. In very light sleep, humps with amplitudes up to $1.00 \mu \mathrm{V}$ were visible in

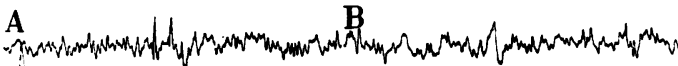

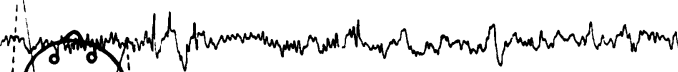

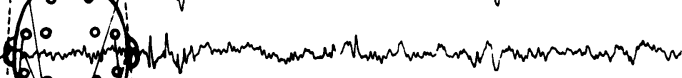

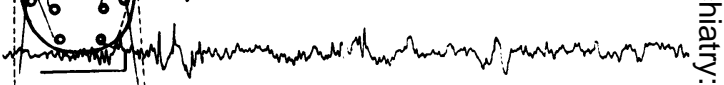

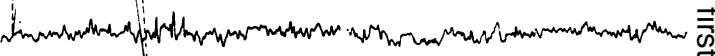

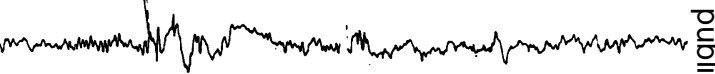

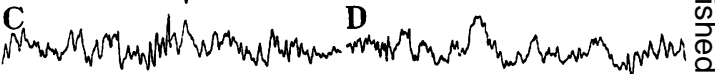

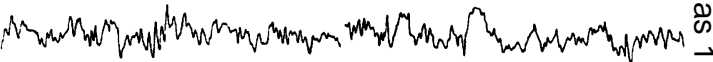

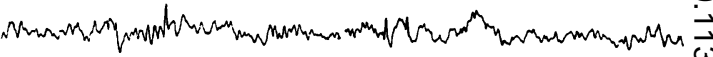

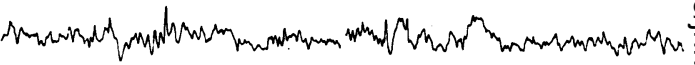

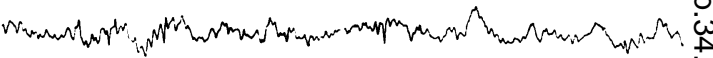

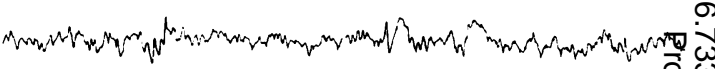

FIG. 6. Sleep EEG of case 1; note infrequent paroxysis and ill-formed sleep patterns. A, B, C, and D indicafe drowsy state, very light sleep, light sleep, and deep sleep $\mathbb{}$ respectively.

bilateral parietal and central areas but most of them $\overrightarrow{0}$ were ill-formed. Fast activity became less marked, $\underset{ }{-}$ while sharp waves were sporadically seen in bilateral parietal, occipital, and temporal areas, often more pronounced on the right side. In light sleep, sigma rhythm (spindle) of 13 to $14 \mathrm{~Hz}$ with relatively high amplitudes up to $100 \mu \mathrm{V}$ appeared with brief dura-음 tion. There were infrequent monophasic and diphasic 2 sharp waves in parietal, occipital, and temporal $\overrightarrow{\overline{0}}$ areas. In moderately deep sleep, the stage of sigma 3 rhythm with irregular slow waves, fast activity was scarcely seen, and paroxysms of spikes, positive spiky waves, and sharp waves occurred sporadically. $\overline{\bar{Z}}$ In deep sleep, large irregular slow waves were seen but not so commonly. Normal-voltage sharp waves $\overline{ }$ of 16 to $18 \mathrm{~Hz}$ could be observed at times in parietal, occipital and temporal regions, maximal in occipital areas. In short, myoclonus epilepsy showed, as a rule, irregular sleep patterns with infrequent 을 paroxysms. Ill-formed humps and sigma rhythm of $D$ brief duration appeared. Furthermore, paroxysms decreased in number as the patient dropped off into sleep. Table 2 shows the number of paroxysms for ${ }^{-r}$ 60 seconds of each stage of sleep. In the drowsy state $N$ the number of paroxysms decreased to one-third $N$ 
TABLE 2

NUMBER OF PAROXYSMS FOR 1 MIN IN EACH STAGE OF SLEEP

\begin{tabular}{lcccr}
\hline Stage & Spike & $\begin{array}{c}\text { Positive } \\
\text { spike }\end{array}$ & $\begin{array}{l}\text { Sharp } \\
\text { wave }\end{array}$ & Total \\
\hline Awake & 5 & 2 & 17 & 24 \\
Drowsy & 3 & 0 & 5 & 8 \\
Very light & 0 & 0 & 7 & 7 \\
Light & 0 & 0 & 3 & 3 \\
Moderately deep & 1 & 1 & 2 & 4 \\
Deep & 0 & 0 & 3 & 3 \\
\hline
\end{tabular}

of the awake state, and in light sleep to one-eighth. This coincides with clinical observations that myoclonic jerks are absent during sleep.

\section{DISCUSSION}

EEGs of myoclonus epilepsy were reported by previous authors and were described as showing continuous severe generalized slow waves interspersed with spikes, polyspikes, and sharp waves (Grinker et al., 1938; Delay, Fischgold, Pichot, and Verdeaux, 1947; Gastaut and Rémond, 1952; Watson and Denny-Brown, 1953; De Ajuriaguerra, Sigwald, and Piot, 1954; Schwarz and Yanoff, 1965). Indeed, EEGs of our second and fourth case showed prominent high-voltage slow waves with or without spikes, but EEGs of the first case at a terminal stage were dominated by a large number of spikes, polyspikes, and sharp waves with less marked slow waves. EEGs of patients of Harriman and Millar (1955) at the terminal stage also showed frequent spikes. Van Heycop Ten Ham and De Jager (1963) reported that EEGs failed to change in the course of illness. But Namba, Ota, and Fukunaga (1966) reported that spikes and polyspikes increased in number at a later stage. In our second case, the EEG recorded one year later showed that spike components of spikewaves increased in amplitude. Considering his mother's EEG, it might be said that spikes would increase not only in amplitude but also in number at a later stage. Previous authors have not referred to the fast activity which we observed in myoclonus epilepsy. It is of interest that this fast activity, marked in centro-parietal areas, became greater at a later stage and in the first case sometimes changed into sharp waves. As to localization, Grinker et al. (1938) concluded that the discharge had its primary focus in the frontal lobes, while Gastaut and Rémond (1952) felt that the discharge centre was in the upper brain-stem or thalamus. Indeed, in our third and fourth cases high-voltage slow waves were marked in frontal areas, but in the first and third cases spikes and polyspikes were widespread and maximal in parietal areas. These findings suggest that discharges originate in the subcortical structures, because spikes are a sign of a primary epileptogenic lesion, as was described by Penfield and Jasper (1954).

It is well known that the EEG of myoclonus epilepsy is activated by photic stimulation, but its mechanism remains incompletely elucidated. Gastaut and Hunter (1950) concluded that photic activation in idiopathic epilepsy was due to an abnormal permeability of the thalamic synapses. The result we obtained was that an increase in number of paroxysms depended not upon the frequency of stimulus but upon the intensity. This forms a striking contrast with the photic driving which appears in normal subjects by stimulation with a frequency of about $10 \mathrm{~Hz}$. Furthermore, no $\alpha$-rhythm was discernible in myoclonus epilepsy except during the very first phase in the second patient of Van Heycop Ten Ham and De Jager (1963). This suggests that there must be a disturbance of the upper brain-stem (the thalamus and its related structures) in myoclonus epilepsy. It seems that impulses from the retina are transmitted to these structures and trigger paroxysmal discharges. Indeed, in cats one of us has obtained photic responses in the upper brain-stem (Kadobayashi, 1970).

As Lennox (1960) pointed out: 'report of sleeprecordings in myoclonus epilepsy has not been encountered.' We had an opportunity to record sleepEEGs in three cases and one of them reached a deep sleep. In our recording the number of paroxysms during sleep was very much smaller than that during the awake state. This may be due to a reduction of reticular activity and of sensory stimuli during sleep. When it is considered that during the awake state impulses from sensory stimuli and from the reticular activating system may cause paroxysmal discharges, decreased sensory stimuli and reduced impulses from the reticular system during sleep would result in producing fewer discharges.

\section{REFERENCES}

Ajuriaguerra, J. De, Sigwald, J., and Piot, Cl. (1954). Myoclonie-épilepsie familiale de type Unverricht. Etude clinique, électro-encéphalographique et anatomique. Presse méd., 62, 1813-1816.

Delay, J., Fischgold, H., Pichot, P., and Verdeaux, G. (1947). L'épilepsie myoclonique de type Unverricht. Étude génétique. Constatations électro-encéphalographiques. Rev. neurol., 79, 430-433.

Gastaut, H., and Hunter, J. (1950). An experimental study of the mechanism of photic activation in idiopathic epilepsy. EEG clin. Neurophysiol., 2, 263-287.

Gastaut, H., and Rémond, A. (1952). Étude électroencéphalographique des myoclonies. Rev. neurol., 86, 596-609.

Grinker, R. R., Serota, H., and Stein, S. I. (1938). Myoclonic epilepsy. Arch. Neurol. Psychiat. (Chic.), 40, 968-980. 
Harriman, D. G. F., and Millar, J. H. D. (1955). Progressive familial myoclonic epilepsy in three families: its clinical features and pathological basis. Brain, 78, 325-349.

Kadobayashi, I., Ukida, G., Kita, K., and Iizuka, R. (1969). An electroencephalographic study on a family of myoclonus epilepsy. Brain Nerve (Tokyo), 21, 1253-1258.

Kadobayashi, I. (1970). Effects of lenticular stimulation on photically evoked potentials in cats. Psychiat. Neurol. jap., 72, 365-377.

Lennox, W. G. (1960). Epilepsy and Related Disorders, Vol. 1. Little, Brown: Boston, Mass.

Namba, M., Ota, T., and Fukunaga, S. (1966). The investigation of electroencephalogram of patients suffering from myoclonus epilepsy (Lafora-type) and its family. Brain Nerve (Tokyo), 18, 1165-1173.
Penfield, W., and Jasper, H. (1954). Epilepsy and the Functional Anatomy of the Human Brain. Little, Brown: Boston, Mass.

Schwarz, G. A., and Yanoff, M. (1965). Lafora's disease. Distinct clinico-pathologic form of Unverricht's syndrome. Arch. Neurol. (Chic.), 12, 172-188.

Unverricht, H. (1891). Die Myoclonie. Deuticke: Leipzig.

Unverricht, H. (1895). Über familiäre Myoclonie. Dtsch. Z Nervenheilk., 7, 32-67.

Van Heycop Ten Ham, M. W., and Jager, H. De (1963). Progressive myoclonus epilepsy with Lafora bodies. Clinical-pathological features. Epilepsia (Amst.), 4, 95-119. Watson, C. W., and Denny-Brown, D. (1953). Myoclonus epilepsy as a symptom of diffuse neuronal disease. Arch. Neurol. Psychiat. (Chic.), 70, 151-168. 